\title{
A PHENOMENOLOGICAL FRAMEWORK FOR THE RELATIONSHIP BETWEEN THE SEMANTIC WEB AND USER-CENTERED TAGGING SYSTEMS
}

\author{
D. Grant Campbell <gcampbel@uwo.ca> \\ Faculty of Information and Media Studies \\ University of Western Ontario \\ London, Ontario N6A 5B7, Canada
}

\begin{abstract}
This paper uses Husserl's theory of phenomenology to provide a model for the relationship between user-centered tagging systems, such as del.icio.us, and the more highly structured systems of the Semantic Web. Using three aspects of phenomenological theory-the movement of the mind out towards an entity and then back in an act of reflection, multiplicities within unity, and the sharing of intentionalities within a community - the discussion suggests that both tagging systems and the Semantic Web foster an intersubjective domain for the sharing and use of information resources. The Semantic Web, however, resembles traditional library systems, in that it relies for this intersubjective domain on the conscious implementation of domain-centered standards which are then encoded for machine processing, while tagging systems work on implied principles of emergence.
\end{abstract}

\section{Introduction}

Since its inception, the World Wide Web has both exceeded the expectations of its detractors, and confounded the expectations of its inventors. The exponential growth of the Web, in its numbers of nodes, the number of its regular users, and its importance as a social, economic and intellectual force in human life has been nothing short of a revolution. And yet, the Web's evolution as a means of browsing pre-fabricated information for purposes of entertainment, enlightenment and e-commerce has, until recently, overshadowed the dreams of its inventor. Tim Berners-Lee originally saw the Web as a forum for collaboration, in which writing to the Web would be as easy as accessing the Web, and in which people could give as easily as they could receive.

In recent years, this dream has finally shown signs of becoming true in a variety of ways: in the rise of weblogs and wikis, which promote the easy creation and modification of Web information; 
in the rise of RSS aggregators which create customized feeds of information according to personal needs and interests; and in the rise of social bookmarking tools which enable users both to mark pages of interest, and to assign verbal tags that describe the facets of interest.

Ironically, Berners-Lee's dream may be coming true at the expense of his own chosen means of bringing it about. The Semantic Web - the brainchild of Berners-Lee and the World Wide Web Consortium - has long professed to be the next generation of Web infrastructure. Semantic Web standards, such as the Resource Description Framework (RDF), the RDF Schema, Annotea, XPath and the Working Ontology Language (OWL), aim to make Web information machineunderstandable as well as machine readable, in the form of context-specific metadata that will enable semantic agents to filter, recombine and present on-the-fly information displays that meet specific needs. But the Semantic Web, which began development close on the heels of the World Wide Web, has yet to reach critical mass, while social tagging systems such as del.icio.us, connotea and citeulike have grown massively in size and popularity.

What is the relationship between social tagging systems and the Semantic Web? Is social tagging merely “mob indexing," as Peter Morville suggests (2005): a transiently popular but unsatisfactory substitute for better, more sophisticated concepts of findability? Or does social tagging represent the successful realization of a better, more useful Web, while the Semantic Web languishes as a failed dream? Are the two on a continuum? Or are they opposed to each other?

\section{Social Tagging vs. Highly-Structured Systems}

Like the Semantic Web, user-centered tagging systems rest on concepts and assumptions that are basic to library classification and description: that information resources require some form of collocation based on someone's decision about the resource's content, its potential usefulness, and its relationships with other resources. Like the Semantic Web, user tagging takes these assumptions and moves them out of the library community, with its technical services departments and its battery of trained cataloguers and indexers. Classification, taken in its broadest sense as the act of gathering like things together in a useful way, can be done by end users, using their own words, and then using web-based digital technologies to assemble their varied decisions together in new and interesting ways. 
Tagging systems, however, differ profoundly from the Semantic Web in the relative simplicity of their methods. The Semantic Web relies on the widespread adoption of information description encoding standards. Information will be described according to the Resource Description Framework, an XML-based method of describing resources which can move from the relative simplicity of RSS feeds to potentially monumental complexity. Information will be described according to metadata standards invoked through namespaces; these different metadata standards can be reconciled through ontologies, facilitating interoperability and cross-domain resource discovery.

The Semantic Web, therefore, has the potential to support systems as complex and highly structured as those in use in libraries today: classification systems, controlled vocabularies, and richly-detailed resource description standards like $A A C R 2$ and the forthcoming Rules for Description and Access. Such complexity is not required; RSS feeds typically use very simple RDF to syndicate information. But the Semantic Web can house such complexity within its standards.

Tagging systems such as del.icio.us make no such claim. The user, upon encountering a resource of interest, can post a description to the tagging system. In addition to thinking up words from scratch, the user can typically select from a set of terms extracted from the text of the resource; from a list of tags that other users, if any, have assigned the resource; or from the list of tags the user has assigned to other resources. As Dave Weinberger (2005) aptly put it, tagging systems do not create elaborate subject trees; instead, they create a rake for raking them up.

This simplicity makes tagging systems very attractive and typical of Web 2.0 culture in their ease of use and rapidity of establishment. In this sense, they achieve what Berners-Lee hoped the Semantic Web would achieve: making Web use as easy as taking out a pen, rather than getting out the lawn mower (Berners-Lee \& Fischetti, 1999). And their rapidity of adoption has suggested to some that the highly structured standards like the Semantic Web have made a catastrophic mistake. Clay Shirky, in his provocative talk, "Ontology is Overrated" (2005), argues that Web standards that call for high structure have made the following miscalculations:

- They failed to realize their own revolutionary implications, and ended up recreating the very systems they were designed to replace, just as the first online travel services 
attempted to recreate travel agencies, only to be swept away by the next generation of simpler systems, such as Expedia.

- They failed to realize that systems which add only a little structure can meet most of our information needs at a fraction of the cost: "it's not," he argues, "that systems with a little structure are better than high-structure systems; only that they are better than systems with no structure at all."

Tagging systems, then, are a cheaper, easier, faster substitute for the elaborate systems designed by libraries and potentially facilitated by the Semantic Web: furthermore, they are more democratic, exercising less overbearing control in the choice of terms and the decisions of which relationships are valid and which are not.

\section{Tagging and Complexity Theory}

Tagging systems have an additional factor that makes them attractive: scalability. Ever since the astonishing success of the Google PageRank system, information professionals both within and outside the library community have been fascinated by systems which actually become better as they are more heavily used. Accustomed as we are to overuse of limited bandwidth, attenuated circulation systems crashing on busy Saturday afternoons, we cannot avoid being mesmerized by BitTorrent downloads, which get faster as more and more people join in and help with seeding the files. Even more fascinating are systems in which relatively simple components doing relatively simple things aggregate to create patterns that become clearer as more components are added, and without central control.

The extraordinary success of the Google PageRank algorithm — which treats each link to a site as a vote in favour of that site, and uses the links as a means of ranking its hits - has given a fresh cachet to theories of complexity and emergence, popularized in such works as Mitchell Waldrop's Complexity (1992) and Steven Johnson's Emergence (2001). In these treatments, the concept of the self-organizing system is transformed into a general belief that the Internet is capable of emerging patterns of organization, similar to that of anthills and neighbourhoods.

By extension, if you let users tag their own resources in their own ways, with their own words, patterns of order will emerge; these patterns will be truer, more convincing, more user-centered, and more useful than the patterns imposed by formal classification schemes. What's more, they 
will acquire greater accuracy and greater sophistication as more and more people use them; schemes like del.icio.us, then, can scale up in ways that conventional organization systems, constrained by limited personnel and limited time, cannot.

Coupled with the genuine fascination inspired by the rich theories and intriguing possibilities of complexity theory, there is a potential for other kinds of fascination, which should probably be avoided. When complexity theory gets translated into information policy, it may take the form of laissez-faire librarianship: sit back, do nothing, and wait for wonderful information patterns to emerge. For cash-strapped politicians struggling to balance principles of universal access to essential services with the costs of creating an information infrastructure, self-organization comes to look like a very handy rationale for abandoning highly-structured (and expensive) systems.

Unfortunately, complexity theory itself suggests that laissez-faire librarianship is a bad idea. If we accept the notion that the Web is a complex, adaptive, evolving system, then Wolfram's findings (1984) suggest that complex systems don't just happen. Some systems freeze into stasis, either immediately or after a very short burst of activity; other systems spin into chaos. Only occasionally does genuinely complex growth take place. We cannot assume that useful information will emerge from systems like del.icio.us, simply by backing off and leaving them alone.

Decisions, then, will be called for: rational, informed decisions about the kind of information environment we wish to create for ourselves. We need a framework for guiding our decisions. How is the power of tagging systems to be balanced against the power (and cost) of more elaborate standards like library cataloguing and the Semantic Web?

Classification researchers have provided us with a wealth of options, drawn from classification theory, philosophy, and critical theory. Recent trends continue to argue for frameworks that allow for multiple meanings and approaches, with greater sensitivity to context and greater amplitude of methods and approaches. Tennis (2005) uses experientialist philosophy to suggest that frameworks for networked information resources must take into account both formal, or structural, and associative, or interpretive sources (79). Mai (2005) suggests that our documentcentered approaches to indexing prevent us from fully recognizing and utilizing important contextual information that emerges in a domain-centered approach (599). Frohmann (2004) uses Nunberg and Wittgenstein to question our assumptions about the permanent meaning of 
documents: meaning that can theoretically be maintained when the technological medium changes (388). Furner (2004) suggests that relevance theory and research has a promising role to play in the development of information theory (445).

This paper is part of a larger project analyzing the Semantic Web in light of Foucault's archaeological theories. In this part of the project, however, I have chosen to look, not through the lens of Foucault, but through the lens of Edmund Husserl, one of Foucault's most important formative influences. And for the remainder of this paper, I will argue that Husserl's concept of phenomenonology, which he articulated for the 1929 edition of the Encyclopedia Britannica, provide a useful insight into the ways in which tagging systems can co-exist alongside more highly structured systems such as the Semantic Web.

\section{Phenomenology}

Phenomenology is frequently set off against empiricism in the selection of research methods: "any effort to understand human behaviour must take into account that humans are cognitive beings who actively perceive and make sense of the world around them" (Palys 1997, 16). As defined by Husserl, phenomenology posits a distinction between outward perception and inward reflection, and our awareness of the external world takes the form of inner manifestations in the consciousness which Husserl calls "phenomena." Husserl did not dispute the existence of the outer world, but he argued that a true unity of the sciences could only emerge through the rigorous establishment of all sciences on the basis of a phenomenological intentionality. This intentionality has three features which are of particular interest:

\subsection{Dual Movement}

Husserl bases his approach to consciousness, being and the psychology of experience in the concept of "reflection": the turning inward of an outward glance. Conscious activity, he argues, focuses upon specific things and not on the psychical experience as such. Psychic activity is the act of reflection. We don't grasp objects "straight out"; rather, we grasp their "phenomena": the subjective experience in which we become conscious of them. Husserl defines this reflective consciousness as "intentionality," in which our intention goes out towards the external object and is then reflected back. 


\subsection{Multiplicities and Unity}

Phenomenology addresses the age-old problem of multiplicities and unity by suggesting that our "unified" intentional concept of an entity has multiple modes of appearance. A die, he claims, can look different, depending on its orientation, and depending on its distance from the viewer. In addition, when we refer to the unified concept of the die, we generally include those undetermined unseen parts, such as the sides that are not facing us. Each of these modes is consciousness of something; but they all create a synthetic and unified awareness.

\subsection{Shared Intersubjectivity}

Most importantly for our purposes, Husserl argues that the psychic process of reflection and intentionality is accessible through the experience of others. Members of a community can share their reflections, thereby ratifying their own experience through matching experiences, as well as encountering new experiences. This process of sharing establishes one's own difference vis-à-vis the community; at the same time, it establishes properties of perception and subjectivity peculiar to the life of the community. This Husserl argues, gives rise to a new task: to render phenomenologically understandable the mental life of the community, and all the intentionalities that pertain to it. For Husserl, every mind has two things:

- An intentional life process that constructs unities of sense directed towards objects; - $\quad$ An experiencing "I" subject: an I-pole at the center for all intentionalities.

A community - whether it is based on geographical proximity, a common objective, or a common domain - is a group of pure persons acting the intersubjective realm of the pure life of the consciousness. Their individual "I-poles" form commonly-held intentionalities that make it possible to interact meaningfully (See Fig. 1). 


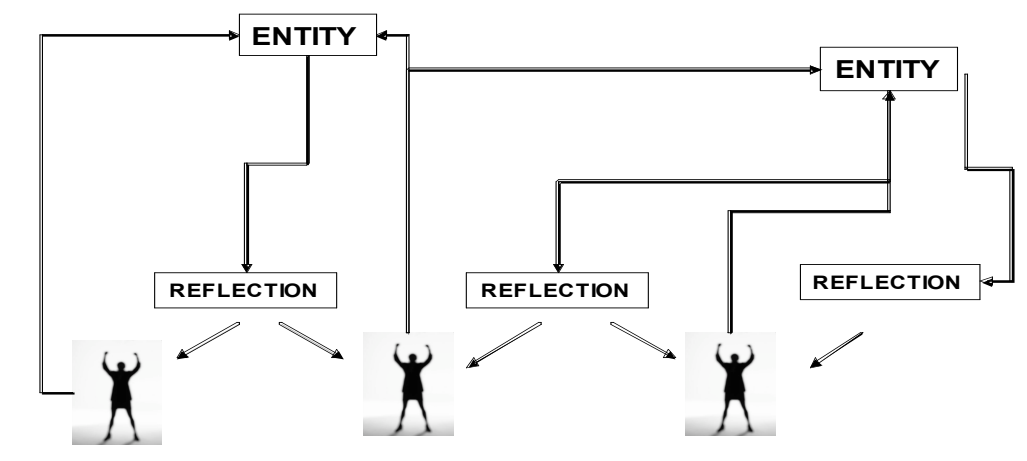

\section{Figure 1: Shared Intersubjectivity}

These three facets of phenomenology provide a useful template for comparing tagging systems to the Semantic Web.

\section{Tagging}

\subsection{The Outward Urge vs. Inward Reflection}

Tagging systems like del.icio.us enable the user to place a posting mechanism on a browser toolbar. At any point in a given task or activity, the user can easily create a del.icio.us bookmark for any website on current view. The act of posting involves a turning of the user's perspective towards reflection, if only with the decision that a particular resource is worth nothing. Upon making that decision, the user then faces a series of options, each of which serves to enhance the reflective integration of the site into the user's domain of interests and concerns. 


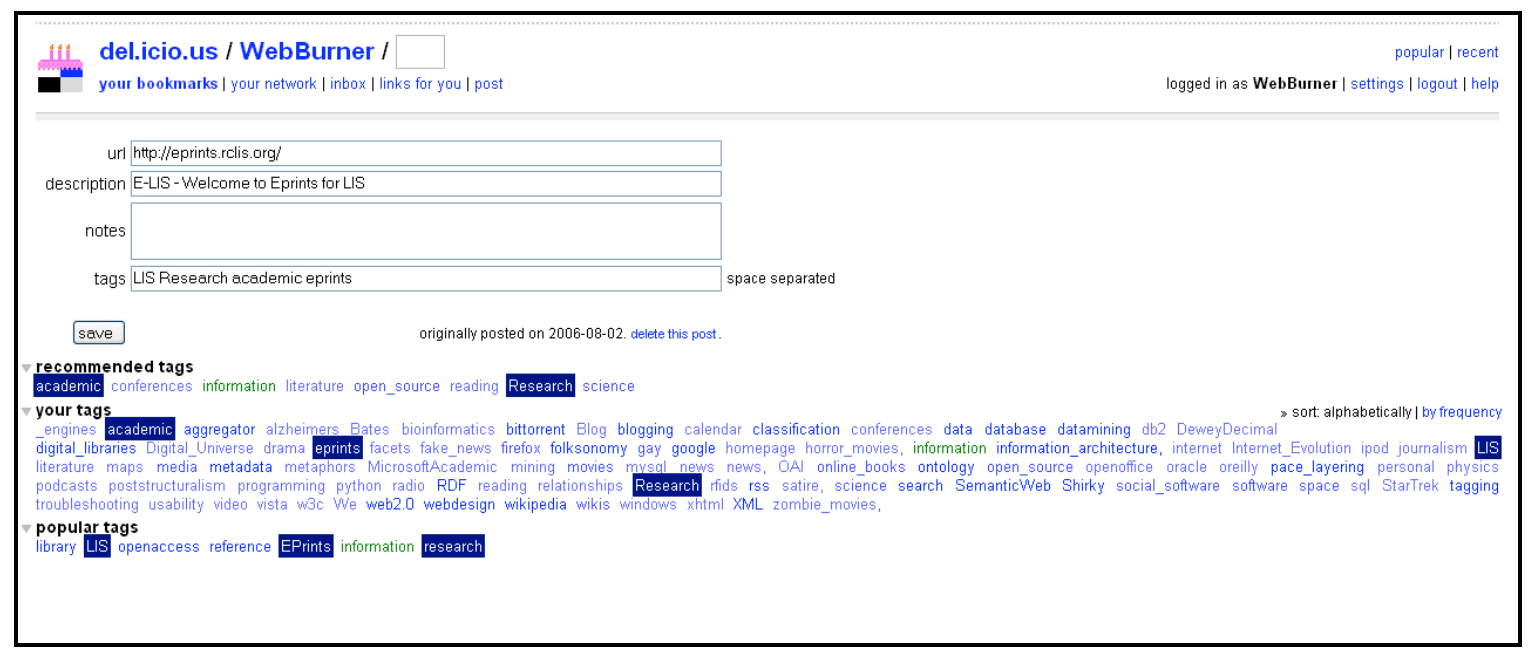

Figure 2: Tagging Options in del.icio.us

As Figure 1 indicates, creating verbal tags is optional; if created, however, the user has the option of entering them manually, or of selecting from up to three sources:

- recommended tags based on how others have tagged the resource;

- tags that the user has used for other documents;

- popular tags.

The tagging system, therefore, encourages the user to integrate his or her reflection of the resource into evolving structures: the user's own tags, those of other users, and those that are the most popular. The bookmarked site, therefore, appears within a context constructed from multiple answers to the question, "why is this important to me?"

\subsection{Multiplicities}

As any librarian knows from experience, documents can be important to users for various reasons. Preliminary analyses of tagging patterns (Kipp \& Campbell, 2006) indicate that the intentionality inherent in tagging embraces multiple modes of representation. To begin with, users are no better than trained indexers at reaching consensus on aboutness: they differ widely in their depth and specificity, their choice of primary topic, phase relations, and so on. ${ }^{1}$ Each

\footnotetext{
${ }^{1}$ For purposes of this discussion, I am ignoring some of the most basic normalization issues, such as different spellings of the same word, and even the uses of different words to mean essentially the same thing.
} 
heavily tagged resource, therefore, emerges as a resource of multiple topics, carrying multiple significance.

In addition, tagging straddles another thorny indexing distinction: between aboutness, as a statement of the essential content of the resource, and meaning as a statement of the resources usefulness in a specific context (Beghtol, 1986, 85). Many of the tags are task-related, rather than subject-related, and the statements of tasks vary from unspecified labels such as "ToRead" and "ToDo" to specific tags such as "paper" or "Support_Group." Because these tags have meaning only for the person who applied the tags, tagging combines issues of both personal and public significance. And because papers eventually get written, and things to do either get done or remain undone, tagging also combines issues of temporary significance with those of enduring significance.

Like Husserl's die, then, a site that is heavily tagged acquires multiple modes, depending on a variety of perspectives, tasks and uses, some of which are evident to all and some only to a few.

\subsection{Shared Intersubjectivities}

In social tagging, these multiple perspectives can be shared, and the sharing enables users to benefit, not just from their own discoveries, but from those of others. In Figure 3, for example, the user interested in "ontology" can retrieve the most popular sites that have been tagged with that term, as well as a list of related tags generated by the tagging patterns of the users:

\begin{tabular}{|c|c|c|}
\hline del.icio.us / popular / ontology & logged in as WebBurner & popular | recent \\
\hline Popular items tagged ontology $\rightarrow$ view yours, all & del.icio.us & $\checkmark$ search \\
\hline $\begin{array}{l}\text { Wikipedia 3.0: The End of Google? save this } \\
\text { first posted by evolvingtrends on } 2006-06-26 \text {... Eaved by } 257 \text { other peopie ( } 57 \text { recently) }\end{array}$ & & \multirow{5}{*}{$\begin{array}{l}\text { related tags } \\
\text { semanticweb } \\
\text { research } \\
\text { toread } \\
\text { tagging } \\
\text { soffware } \\
\text { wiki } \\
\text { owl } \\
\text { knowledge } \\
\text { web } \\
\text { search } \\
\text { semantic }\end{array}$} \\
\hline $\begin{array}{l}\text { OpenCyc. org save this } \\
\text { first posted by badzen on 2004-02-22 ... Baved by } 440 \text { olter people ( } 51 \text { recently) }\end{array}$ & & \\
\hline $\begin{array}{l}\text { SIMILE Project save this } \\
\text { first posted by badzen on } 2004-06-14 \ldots \text { Eaved by } 602 \text { other people ( } 49 \text { recently) }\end{array}$ & & \\
\hline $\begin{array}{l}\text { Shirky: Ontology is Overrated -- Categories, Links, and Tags save this } \\
\text { first posted by bop on 2005-05-16 ... }\end{array}$ & & \\
\hline $\begin{array}{l}\text { Swoogle save this } \\
\text { first posted by bibo on } 2004-09-12 \ldots \text { baved by } 423 \text { other people ( } 47 \text { recently) }\end{array}$ & & \\
\hline $\begin{array}{l}\text { Shirky. Ontology is Overrated -- Categories, Links, and Tags save this } \\
\text { first posted by mlamb on } 2005-05-16 \ldots\end{array}$ & & \\
\hline
\end{tabular}

Figure 3: Popular sites tagged "Ontology" 
Individual users, each with specific tasks, needs and objectives, act as Husserl's "I-poles": their tags enable them to establish commonly-held intentional relationships with resources that make resource sharing interesting and meaningful for others. The now-ubiguitous tag clouds of tagging systems establish domains of intersubjectivity that make interaction and knowledge-building possible.

\section{The Semantic Web}

\subsection{The Outward Urge vs. Inward Reflection}

The Semantic Web also works on a combination of outward encounters with resources and inward reflection that integrates those resources into a working structure. However, the sequence of these activities, and the distribution of the tasks involved, can be very different. Because the Semantic Web, among other things, aims to facilitate access to the "deep web"- data that is contained in relational databases with a Web gateway-much of the data that gets encoded into RDF has already been subjected to the intense scrutiny and normalization of database design. Similarly, web agents that search the Web for RDF-encoded data will be finding materials that have already been reflectively encoded, by the creators or by others, in anticipation of specific user needs.

This encoding can potentially do more than give the resource structure for highly-granular retrieval; it can also encode the potential concerns that the user will bring to the document, including:

- Which of my questions can this resource answer?

- How trustworthy is this resource?

- What is its relationship with other resources?

In the following example, a Web resource on Alzheimer's disease has been marked up in simple RDF with Dublin Core metadata; in addition, an answer contained in the site to a user's potential question has been similarly encoded: 
<rdf:Description rdf:about="http://www.alzheimer.ca/english/haveAD/learning.htm"> $<$ dc:title $>$ I Have Alzheimer's Disease: Learning About the Disease $</$ dc:title $>$ $<$ dc:creator $>$ Alzheimer Society of Canada $</$ dc:creator $>$ $<$ dc:publisher $>$ Alzheimer Society of Canada $</$ dc:publisher $>$ $<$ dc:identifier $>$ http://www.alzheimer.ca/english/haveAD/learning.htm $</$ dc:identifier $>$ $<$ dc:description $>$ frequently asked questions $</$ dc:description $>$ $<$ dc:format $>$ html $/$ text $</$ dc:format $>$ $<$ dc:relation $>$ http://www.alzheimer.ca/english/haveAD/intro.htm $</$ dc:relation $>$ $<$ dc:language $>$ en $</$ dc:language $>$ $<$ dc:date $>2005 / 10</$ dc:date $>$

$</$ rdf:Description $>$

$<$ rdf:Description rdf:about="gm0002" $>$

$<$ gcg:question $>$ What is Alzheimer's disease? $<$ gcg:question $>$

$<$ gcg:audience $>$ non-professional $<$ gcg:audience $>$

$<\mathrm{dc}$ :text $>$ Alzheimer's disease is not a normal part of aging. Symptoms include having difficulty remembering things, making decisions and performing tasks. These changes can affect the way a person feels and acts. There is currently no way to stop the disease but there is hope that research will find a cure. $</$ dc:text $>$

$<$ dc:relation>http://www.alzheimer.ca/english/haveAD/learning.htm $</$ dc:relation $>$ $</$ rdf:Description $>$

An RDF data collection, compiled by reference librarians, could conceivably create a resource in which a user, when posing a typical question, receives a specific answer, as in Figure 4. 


\section{What causes Alzheimer's Disease?}

The majority of people with the disease have a common form called Sporadic Alzheimer's Disease. Researchers are trying to determine what role heredity plays in this form.

A very small percentage of people have an inherited form of the disease. This rare form is called Familial Autosomal Dominant Alzheimer's Disease. In certain families, it passes directly from one generation to another. Having more than one family member with Alzheimer's disease does not necessarily mean that your family has the inherited form.
Source:

Title:

I Have Alzheimer 's Disease:

Learning About the Disease

Creator:

Alzheimer Society of Canada

Publisher:

Alzheimer Society of Canada

Date:

2005/10

\section{Figure 4: Potential Display using RDF Data}

This resource, however, does not rest on serendipitous discovery of other people's intentionalities. Instead, it would be the result of information professionals reflecting upon the needs of their users, and embedding that reflection in an RDF framework.

\subsection{Multiplicities}

The Semantic Web was envisioned as a means of collaboration, and continues to attract interest as a means of cross-domain resource discovery. Like tagging systems, therefore, it rests on the assumption that there are multiple ways of looking at the same subject, multiple uses for the same information, and multiple terms to describe the same concept. However, the Semantic Web anticipates that these differences will ultimately undergo significant normalization in the form of domain-specific metadata sets, established by namespaces, and by ontologies that will reconcile different metadata sets to enable a system to retrieve data across different domains.

The Semantic Web, therefore, locates multiplicity not at the level of the individual user, but at the level of individual domains to which the users will presumably belong. The users of a resource 
on Alzheimer's disease, for instance, could be separated into lay readers and health professionals, each of which would have its own metadata set, reconciled at the ontology level.

\subsection{Shared Intersubjectivities}

In this sense, the Semantic Web paradigm resembles the assumptions of tagging systems, which create intersubjectivities by gently suggesting popular and widely-used tags to the user. While the user should theoretically be free to add whatever tags are personally meaningful, no integration into a whole can be achieved without some measure of duplication, and some multiple uses of the same tags. The Semantic Web is more aggressive in its promotion of domain consensus: the user will hopefully belong to some group that has established, for its own users, a meaningful set of entities and terms, and will use those terms.

The intersubjective domain created by the Semantic Web, therefore, rests upon painstaking and precise work by information providers and information intermediaries. It emerges, not from the tag clouds, but rather by the creation and use of namespaces, of ontologies that reconcile different namespaces, of crosswalks between different metadata systems, and the careful encoding of data to make it accessible.

\section{The Semantic Web vs. Social Tagging Systems}

If we compare the Semantic Web to social tagging systems within this phenomenological framework adapted from Husserl, we can envision a new relationship between the two, based not on succession but on complementary purposes. In this relationship, tagging systems enable the emergence of an intersubjective consensus on important entities and relationships based on loose structure and high flexibility. The patterns of this intersubjective realm are unstructured, timesensitive and inconsistent; they are also, in their simplicity and flexibility of implementation, useful, rapidly-evolving and surprising.

The Semantic Web presents an instersubjective realm which is constructed far more painstakingly, far more slowly, and far more methodically. As such, it resembles traditional library systems in its emphasis on high structure and domain consensus. The patterns of the Semantic Web are based on rational and deliberate modeling and encoding. They may be more 
cumbersome and less adaptable than the patterns of social tagging; but they may also be more stable and enduring building blocks of a Web -based information infrastructure.

\section{Conclusions: Limitations, Promises and Dangers}

This analysis has numerous limitations, to say the least. I have oversimplified the complex diversity of both Semantic Web applications and tagging systems: as tagging culture continues to evolve and as the Semantic Web's destiny becomes clearer, other authors will fill in the enormous gaps. We also need a good deal more empirical research on tagging trends, and I hope to make use of that in the future.

Perhaps the most painful limitation is the glib equation of consciousness, as Husserl envisions it in his work, with the Web as a collective mind, in which outward and inward movement, phenomena and intentionalities are manifested in Web resources and Web behaviour. I can only hope that the equation, glib as it may be, has suggested a better and more adaptable way of viewing the relationship between tagging and the Semantic Web.

I feel this the more strongly, because the Semantic Web gets less attention in library science these days than Web 2.0 technologies such as tagging and blogging. And while I recognize the appeal of these technologies for libraries, I also suspect that the Semantic Web provides an opportunity for libraries to play a more enduring, and ultimately more useful role in the future of information. The Semantic Web, with its high structure and its elaborate tagging, offers an alternative to the laissez-faire approach to information, and enables us to make rational, conscious decisions about the fundamental values and relationships — social, cultural and intellectual — that we consider essential in our information environment.

With this promise, however, comes a danger: a danger that tagging systems could potentially alleviate. Because the Semantic Web is based on decisions that are machine-understandable, and not just human-understandable, they will be built into the technical infrastructure of the Web. As such, they run the serious risk of becoming a priori assumptions that govern all uses of the system: invisible, inevitable, inescapable. The surprising, even absurd patterns that emerge through social tagging will form a necessary counterpoint to this infrastructure, and hopefully encourage us to revisit those structures and revise them when necessary. 
Campbell, D. G. (2006). A phenomenological framework for the relationship between the semantic web and user-centered tagging systems. 17th Annual ASIS\&T SIG/CR Classification Research Workshop, 1-17. doi: 10.7152/acro.v17i1.12489

17th SIG/CR Classification Research Workshop, November 4, 2006

\section{Acknowledgements}

The author gratefully acknowledges the assistance of the following persons: Margaret Kipp, Karl Fast, Graham Maclean and Catherine Baird. Funding for this research is generously provided by the Social Sciences and Humanities Research Council of Canada.

\section{References}

Beghtol, C. (1986). Bibliographic classification theory and text linguistics: Aboutness analysis, intertextuality and the cognitive act of classifying documents. Journal of Documentation 42: 84113.

Berners-Lee, T., with M. Fischetti. (1999). Weaving the Web: The original design and ultimate destiny of the World Wide Web. San Francisco: HarperCollins.

Frohmann, B. (2004). Documentation redux: Prolegomenon to (another) philosophy of information. Library Trends 52 (3): 387-408.

Furner, J. (2004). Information studies without information. Library Trends 52 (3): 427-447.

Husserl, E. (1929). Phenomenology. Encyclopedia Britannica. Reprinted in The continental philosophy reader, ed. R. Kearney and M. Rainwater. London: Routledge, 1996: 15-22.

Johnson, S. (2001). Emergence: The connected lives of ants, brains, cities, and software. New York: Scribner.

Kipp, M., and Campbell, D. G. (2006). Patterns and inconsistencies in collaborative tagging systems: an examination of tagging practices. ASIST 2006 Proceedings. In press.

Mai, J.-E. (2004). Analysis in indexing: Document and domain centred approaches. Information Processing and Management 41: 599-611.

Morville, P. (2005). Ambient findability: What we find changes who we become. Cambridge, MA: O'Reilly. 
Palys, T. (1997). Research decisions: Quantitative and qualitative perspectives, 2nd ed. Toronto: Harcourt Brace.

Shirky, C. (2005). Ontologies is overrated. IT Conversations. 1 MP3 file. Retrieved from http://www.itconversations.com/shows/detail470.html.

Tennis, J. (2005). Experientialist epistemology and classification theory: Embodied and dimensional classification. Knowledge Organization 32(2): 79-92.

Waldrop, M. (1992). Complexity: The emerging science at the edge of order and chaos. New York: Touchstone.

Weinberger, D. (2005, February). Taxonomies to tags: From trees to piles of leaves. Release 1.0. Retrieved from http://www.edventure.com/release1/abstracts.php?counter=4579726.

Wolfram, S. (1984). Computer software in science and mathematics. Scientific American September: $188-203$. 